\title{
Dilemmas of a psychiatrist in a developing country
}

\author{
Sa'ad B. Malik
}

It was in early 1984 when, after receiving my basic postgraduate training in the UK, I returned to my home country. Pakistan. As I began to settle down in my professional work I soon realised that I was facing problems on more than one front. Whereas some of these I have been able to overcome with time, others remain largely unresolved. Psychiatrists from the developing world who receive their training in the West and subsequently return to their home countries may be facing similar dilemmas. Here I would like to share some of my own.

Pakistan is a developing country. With a population of over 133 million and less than 200 psychiatrists, the mental health services remain inadequate and underdeveloped. At present. psychiatric services are limited to the major teaching centres only. The bulk of the population $(70 \%)$ that lives in the rural setting is thus deprived of any form of modern psychiatric care. Almost all psychiatric manifestations are considered to be the result of possession by evil spirits or the effect of magic spells and the local faith healers are always there to confirm this. While some of the methods used to drive away these 'evil forces' may be harmless (e.g. reciting holy verses) there are others that are truly horrific. Some of the latter include beating, chaining the patients to trees around holy shrines and performing blood letting procedures. These practices, tragically, continue unabated even today.

\section{Coping with patients from a different socio-cultural background}

It did not take me long to realise that my entire training in psychiatry was based on the Western model, more specifically the British one. Using the knowledge derived from Western-based books and journals, seeing patients and working with colleagues who happened to be all British created no problems when working in the UK. Faced with patients from my own cultural background I found myself rather ill equipped. For example, I felt quite helpless when I had to first examine a lady wearing a 'Hejab' (face cover with a veil). I realised that such patients felt uncomfortable lifting the vell before a male doctor as they also did of being interviewed alone. The accompanying husband also usually disapproved of any attempt on the part of the doctor to see the patient on her own. To make matters worse, in many situations the husband acted as the interpreter as the wife often spoke a different dialect or language.

Since patients come from very diverse backgrounds, a psychiatrist here has to often rapidly switch to an entirely different language, manner and behaviour in order to make the patient at ease. For some patients, and this includes the upper class of this society, conversing in English. shaking hands, greeting the patients at the door are all very well received, there are others, particularly from the rural areas, for whom this approach is totally foreign and positively uncomfortable. Rural patients may perceive the doctor as a figure of authority, whom they look to for help and guidance, and variation from this role can make them extremely uncomfortable, and undermine confidence in the treating doctor. I still remember when, during my early days of practice, a patient from a remote northern area of the country found my standing up to greet every patient rather awkward and incomprehensible.

\section{Personal beliefs and attitudes of local psychiatrists}

Dealing with local cultural beliefs is another difficult area. Belief in the existence of magic, and possession by evil spirits is almost universal. Over the years I have come to respect the traditional beliefs of my patients, and do not entirely discard them as meaningless. I feel I have no right to pass judgement on these in the same way that I have no right to do the same on. for example, the sexual life styles of some patients in the West which do not find acceptance with my own personal, cultural and moral values.

Many psychiatrists here are practising Muslims, with religion playing an important role in their everyday life. Their religious beliefs 
not infrequently come into direct clash with 'unorthodox' Western concepts on which their only knowledge of psychiatry is based. Having interviewed a large number of eminent psychiatrists and psychologists in the country, I was unable to discover any uniformity of opinion on a number of issues where religion, culture and the patient's distress come into direct conflict with each other. Some of these include the problems of dealing with homosexuality, masturbation, use of sex education in schools and management of sexual dysfunctions in unmarried individuals (in a culture where premarital sex is strictly prohibited). These are all serious issues and there are certainly many more. In a culture where a desperate patient may take his life as he is unable to cope with his homosexuality, it can be disturbing for his psychiatrist who may feel helpless and lost in addressing such matters. At present, it seems to be the personal outlook of the treating psychiatrist which determines what treatment the patient receives. A psychiatrist with a religious and conservative approach would seriously condemn the deviant sexual tendencies of a patient, reminding him of the eternal damnation in hell and urging him to be more vigorous with his religious rituals. A liberal clinician is likely to introduce him to any relevant self-help support groups. Yet another may have no hesitation in writing out a long prescription of antidepressants and anxiolytics to 'cure' the malady.

\section{Administrative problems}

I often envy my colleagues in the West who have the facility to see a limited number of patients in their regular out-patient clinics. This undoubtedly ensures good quality care. In a developing country like my own the out-patient clinics for psychiatric patients often present a scene of chaos. There are no fixed catchment areas and patients can present from any region of the country. Further, there are no fixed appointments and all patients have to be attended to on the same day, as a good number have travelled long distances with no place to stay overnight. While on the one hand there are usually over 40 patients to be seen in less than four hours, on the other hand there is a desperate effort on the part of the psychiatrists to maintain standards for good professional work. Unable to cope, most psychiatrists compromise on the latter. I have to face one of my biggest dilemmas when after teaching my postgraduate students the importance of the one hour psychiatric interview, I expect them to see these 40 patients in less than four hours. The situation becomes increasingly worse in the heat of summer months when drenching in sweat from head to toe one has to interview patients in a hot overcrowded room and still display the accurate empathy, non-possessive warmth and genuineness' of a good therapist.

Shortage of staff, space and finance are aggravating factors. In most cases the patients are too poor to buy the prescribed drugs. When writing a prescription, apart from considering various clinical and pharmacological aspects relating to the drug, considerable thought has to be given to the financial state of the patients to ensure that they will be able to purchase the drug. This is in striking contrast to the situation in the West where the current major emphasis is to prescribe drugs with the minimum side-effects to ensure safety and compliance. No one would disagree with that. My dilemma is that I have to select the cheapest drug available, in many cases, compromising on side-effects. The choice therefore is not between an older tricyclic antidepressant or a new selective serotonin reuptake inhibitor. The choice is between some treatment or no treatment at all.

\section{Updating knowledge}

A good psychiatrist needs to keep abreast of developments in the field and we in the developing world struggle to do this. One has to keep in touch with the latest in all branches of psychiatry. The sub-specialities are almost non-existent and so all varieties of patients ranging from those that would normally fall in the domain of child psychiatry, psychogeriatrics, forensic psychiatry or rehabilitation have all to be catered for.

There are also national and international conferences held every one to two years when a large number of psychiatrists from home and abroad participate in the proceedings. These are invariably held in the comfortable and impressive setting of some five star hotel and provide an occasion for everyone to develop the 'right' contacts and opportunity to enjoy the hospitality of the East. Lavish sums of money are spent on these expensive get togethers which sadly contribute little in promoting the cause of psychiatry in poorer countries.

\section{Concluding remarks}

As a large number of trainee psychiatrists from the developing world continue to receive postgraduate training in Britain and other Western countries, I would advise them to work hard and maximally benefit from this unique opportunity. This basic training should provide the solid foundation for practice when they return to their respective countries. However, they will need to be aware that they will have to modify their 
methods and concepts to suit the locally prevalent conditions. They must at all costs keep up with the high standards of professional work that the MRCPsych expects of them, but at the same time be prepared to put up with the stresses and hardships that will make this task difficult, though not entirely impossible.
At the end of the day, judging by my own struggle over the past 14 years, it can turn out to be a very satisfying experience.

Sa'ad B. Malik. Professor of Psychiatry, The Postgraduate Medical Institute, Birdwood Road. Lahore, Pakistan

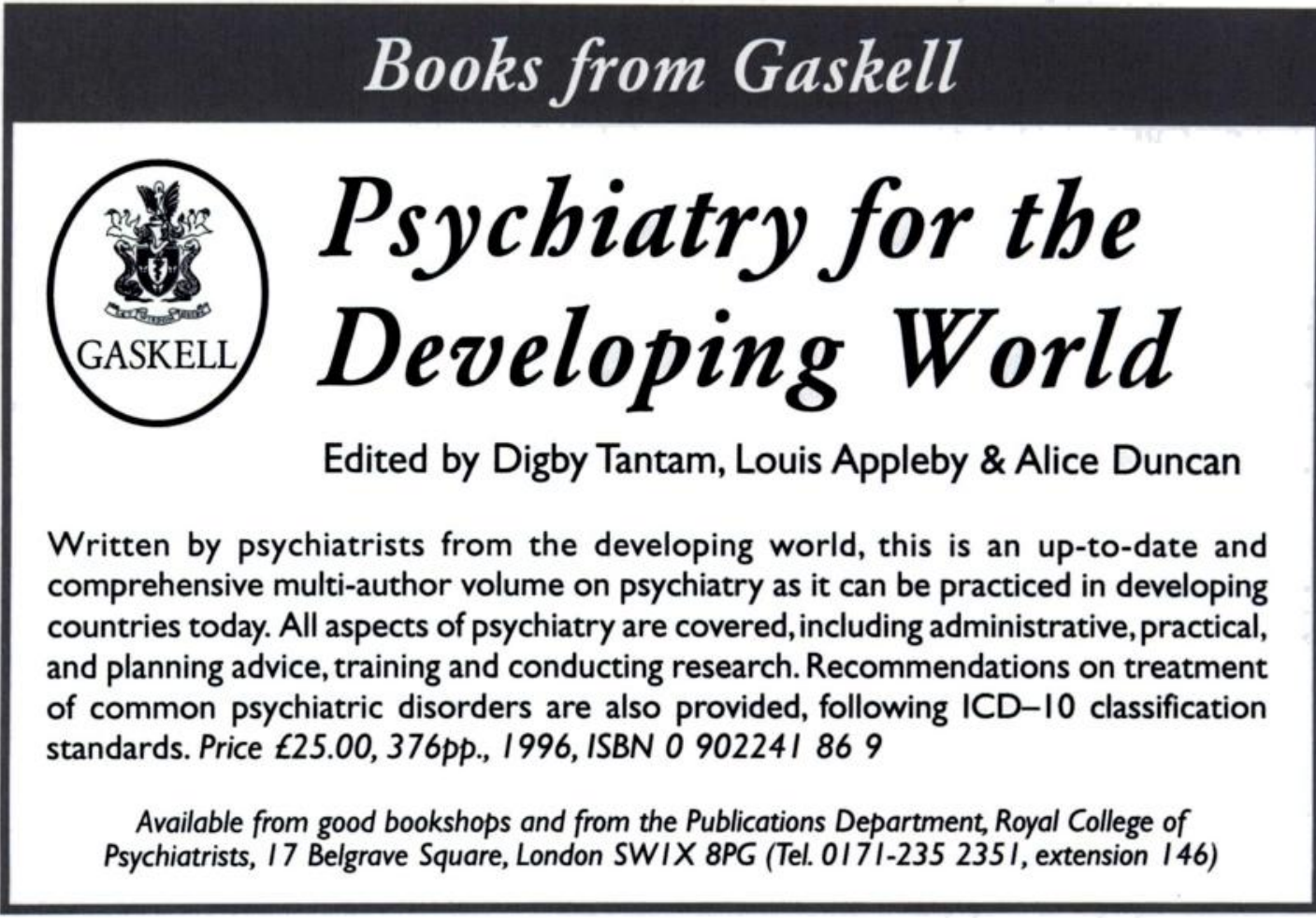

